\title{
Conducting and Electric Force Microscopy of CNT Polymer Nanocomposites: Understanding the Connectivity of the Percolative Network
}

\author{
A. Trionfi, ${ }^{1, *}$ W. P. Hsu, ${ }^{1,+}$ A. Sellinger, ${ }^{2}$ R. A. Vaia ${ }^{2}$ \\ ${ }^{1}$ Sandia National Laboratories, Albuquerque, New Mexico 87185, USA \\ ${ }^{2}$ Air Force Research Laboratory, Wright-Patterson AFB, Ohio 45433, USA \\ * current address: CNA, 4825 Mark Center Drive, Alexandria, VA 22311 \\ ¥ current address: University of Texas at Dallas, Richardson, TX 75080
}

Polymer-carbon nanotube (CNT) nanocomposites are utilized in a diverse array of electrical applications, ranging from electrostatic discharge coatings and EMI shielding of signal cables, to transistors, memory elements, sensors, and transparent contacts. Notwithstanding, a solid understanding of the relationship between processing methods, the architectural details of the carbon-nanotube percolative network, and the macroscopic electrical performance, such as I-V linearity and broadband impedance, is lacking. Thus, in many instances, predictability, reproducibility and therefore manufacturability are limited.

Key to establishing these relationships are techniques to image and measure the impact of nanoscopic features, such as the local distribution of current, charge, CNTs and CNT-CNT contacts, on the macroscopic electrical response. We will discuss two methods for investigating these characteristics in greater depth.

First, the spatial distribution of current paths within CNT polymer nanocomposites can be quantified using conducting-tip atomic force microscopy (C-AFM) and electric force microscopy (EFM). Almost all processing approaches for polymer nanocomposites result in a different distribution of CNTs near the sample surface relative to that within the bulk. These structural heterogeneities arise from numerous factors, such as topological packing constraints of rods near a boundary, settling of filler particles, hydrodynamic flows, and drying induced stresses. Details of the surface structure have substantial impact on the formation of electrical contacts, and thus measurement of macroscopic characteristics. The initial CNT distribution underneath the processing-induced, polymer-rich skin layer can be quantified with EFM by modeling the probe-sample geometry as a series of capacitors (Fig 1) [1]. Connectivity of the CNT to a ground plane can also be qualified with regard to local charge accumulation on the CNT at relatively large tip-sample voltages (Fig 1) [1]. Complementary, C-AFM reveals that electrical transport is solely carried by the CNT networks and the areal distribution of high-current regions at the sample surface depends critically on processing (Fig 2) [2]. The non-uniform distribution of current along the length of some CNTs suggests the presence of heterogeneously adsorbed polymer around the CNTs. Two probe conductivity measurements, where the C-AFM tip is contacting a single constituent conducting particle, show that both Ohmic and non-Ohmic pathways exist in the conducting network of the nanocomposite; the later implying tunneling transport may also be present, especially near the percolation threshold [2].

Second, the geometric critical exponent, $\beta$, associated with the percolation probability, (the probability a metallic filler belongs to the conducting network) can be experimentally measured using C-AFM and an oxygen plasma etching technique that controllably removes processing induced 
heterogeneities to reveal representative cross-sections of the bulk CNT network [3]. From the conducting areal density, $\beta \approx 1$ and $\mathrm{t}$ (the exponent for bulk conductivity) $\approx 3$ was found for polyimidecarbon nanofiber nanocomposites. These values are consistent with the predictions for the Bethe lattice and are larger than the values predicted in the 3D lattice percolation model. Such measurements imply that the underlying structure of the network is more complex than a simple random network; and thus are crucial in understanding the robustness of the overall network to external perturbations or failures at CNT-CNT junctions.

With careful sample preparation, conducting probe microcopies are proving to be uniquely suited to investigate correlations between nanoscopic morphology and physical properties; and thus greatly enhance our knowledge of the most important nanoscale features that determine macroscopic properties [4].

\section{References}

[1] A.T. Sellinger, S. Deshmukh, Z. Ounaies, S.N. Kim, R.A. Vaia, Proc. SPIE, (2010) (in-press)

[2] A. Trionfi, D.A. Scrymgeour, J.W.P. Hsu, M.J. Arlen, D. Tomlin, J.D. Jacobs, D. H. Wang, L.-S. Tan, R.A. Vaia, J Appl. Phys. 104, 083708 (2008)

[3] A. Trionfi, D.H. Wang, J.D. Jacobs, L.-S. Tan, R.A. Vaia, J.W.P. Hsu, PRL 102, 116601 (2009)

[4] The authors would like to thank D. H. Wang, J. D. Jacobs and L.-S. Tan for synthetic support. The effort was funded in part by the Air Force Office of Scientific Research, Air Force Research Laboratory Materials and Manufacturing Directorate, and the U.S. Department of Energy, Center for Integrated Nanotechnologies, at Los Alamos and Sandia National Laboratories. Sandia National Laboratories is a multiprogram laboratory operated by Sandia Corporation, a Lockheed-Martin Co., for the U.S. Department of Energy under Contract No. DE-AC04-94AL85000

Figure 1. Polymer rich skin layer of a 2 vol\% SWNT-polyimide (CP2) nanocomposite [1]. AFM tappingmode (a) height $\left(\mathrm{V}_{\text {tip }}=0 \mathrm{~V}\right)$, (b) EFM $\left(\mathrm{V}_{\text {tip }}=5 \mathrm{~V}\right)$, and (c) height $\left(\mathrm{V}_{\text {tip }}=10\right.$ $\mathrm{V})$ images. The z-scale contrast associated with the SWNTs in $\mathrm{c}$ is a result of charge being drawn to the region under the biased tip from a grounded surface electrode in electrical contact with the percolative network. The AFM compensates for the additional electrostatic force on the tip by backing away from the surface to re-establish the cantilever's oscillation amplitude, subsequently inducing false height contrast in the image. Consequently, this enables only the buried SWNTs in electrical contact with the electrode to be visualized.

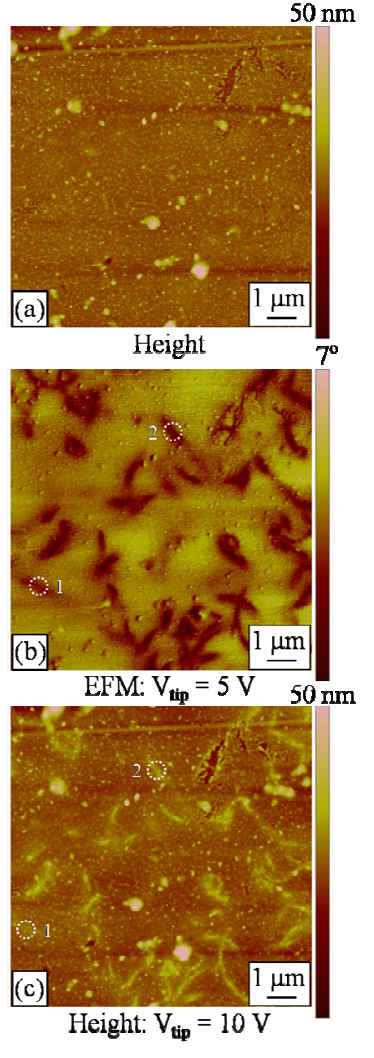

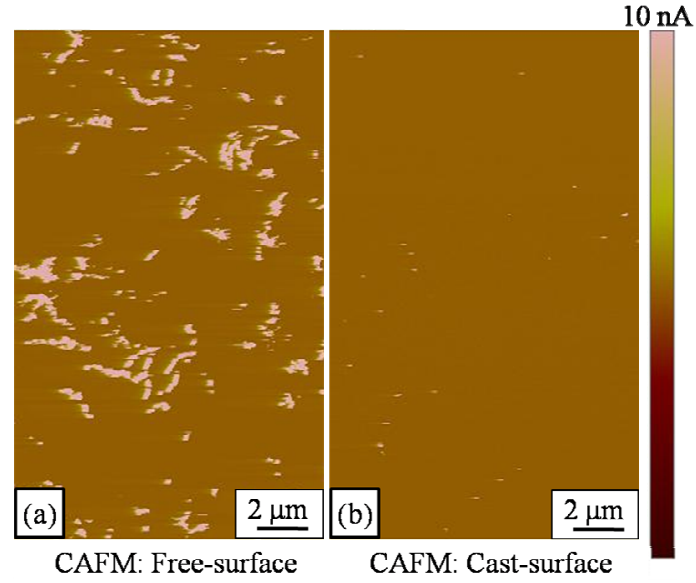

Figure 2. CAFM of the (a) free and (b) cast surfaces of a 2 vol\% SWNT-polyimide (CP2) PNC film. Current carrying features are displayed in white, with the surrounding polymer dielectric in brown. In each case, the current was drawn from the sample base to the tip through the bulk of the percolated film. The minimal contrast in b corresponds to areas where SWNTs are protruding through the polymer skin layer. The areal density of conducting regions reflect the processing induced differences in the electrical properties of the surface. 